\title{
Bargaining in Patent Licensing with Inefficient Outcomes
}

\author{
Yair Tauman* Yoram Weiss ${ }^{\dagger}$ Chang Zhao
}

\begin{abstract}
A monopoly incumbent faces an outside innovator who holds a new technology which has no industrial value to the monopolist but allows a profitable entry. The monopolist is willing to pay for the IP of the technology more than any entrant, in an attempt to limit entry. Still, the innovator may sell a number of licenses before bargaining with the monopolist, even though this will reduce the "bargaining cake". The sale of licenses will credibly increase the innovator's threat on the monopolist, as it increases the number of licenses the innovator will sell in case the bargaining fails.
\end{abstract}

JEL codes: $\mathrm{C} 71, \mathrm{C} 72, \mathrm{C} 78, \mathrm{~L} 13$

*Stony Brook University and The Interdisciplinary Center, Herzliya. Email address: amty21@gmail.com ${ }^{\dagger}$ Tel-Aviv University. Email address: weiss@post.tau.ac.il

${ }^{\ddagger}$ Tel-Aviv University. Email address: zhaochangtd@gmail.com 


\section{Introduction}

Consider a market with a monopoly incumbent firm who has an incentive to fight the introduction of the new technology in an attempt to prevent the loss to his existing profit. In the classical work of Gilbert and Newbery (1982) (GN hereafter), it is shown that a monopolist has an incentive to maintain its monopoly power by patenting new technologies to preempt potential competition, leading to patents that are neither used nor licensed to others (shelving). GN show that this happens if the cost of preemptive patenting is less than the profits gained by preventing entry.

In the presence of outside labs, it is not always the case that the monopolist is aware of all potential competing technologies. In this paper we study such scenario where the monopoly faces an outside innovator who holds the patent of a new technology which allows a profitable entry and as a result will hurt the incumbent's profit if licensed to new entrants. The innovator can either sell his intellectual property to the incumbent or license it to a number of new entrants (or both). To sharpen our analysis we assume that the new technology has no industrial value to the monopolist and whenever the incumbent obtains the new IP he prefers not to use it. The issue is, simply, under what conditions the innovation will not be shelved? If any new entrant obtains a license, then, of course, the innovation will be put to work and shelving does not occur.

Clearly, the incumbent is willing to pay for the IP, even though he does not plan to use the innovation, only if by obtaining the IP he can exclude (or limit) others from using it. We assume that upon reaching an agreement, the innovator and the incumbent will sign a contract which transfers the entire patent right to the incumbent firm. In particular, the incumbent can sell additional licenses to new entrants as he sees fit 1 .

Obviously, if the innovator's payoff from selling licenses to entrants is higher than the monopolist's willingness to pay for preventing entry, entry will occur and the new technology

\footnotetext{
${ }^{1}$ It is obvious that if there are no entrants in the market, the incumbent after obtaining the IP is best off shelving it. If, however, there are already several entrants in the market when he obtains the IP, he may find it beneficial to sell additional licenses. This happens, for instance, in a Cournot market with linear demand.
} 
will be put to work. But even if the monopolist's willingness to pay for preventing entry is higher than the innovator's payoff from selling licenses to entrants, under certain parameters and contrary to the case discussed in GN, entry occurs with certainty. A credible damage of the new technology on the incumbent is typically higher when the innovator sells a few licenses to entrants before bargaining with the incumbent firm. Such action of the innovator, although it brings competition into the market and reduces the total "cake", it increases the bargaining position of the innovator as it increases the incumbent's willingness to pay. To clarify this point, suppose firms compete à la Cournot with a linear demand function. If the innovator approaches the incumbent immediately (without selling any licenses to entrants) and the bargaining fails, the innovator is best off selling 2 licenses. If, however, the innovator sells one license before approaching the incumbent and the bargaining fails, he is best off selling another 3 licenses. In the first scenario if no agreement is reached, the incumbent competes with 2 entrants while in the second scenario he faces 4 competitors. The incumbent has higher incentive to compromise in the later case.

There is another reason why selling licenses before bargaining with the incumbent may be beneficial for the innovator. Bargaining with the incumbent may take significant amount of time ${ }^{2}$ and selling a few licenses prior to the bargaining is the only way the innovator can extract revenues during the bargaining period.

One example that resembles our model (though not perfectly) is the k-cup industry. In the k-cup industry, Green Mountain Coffee Roasters (GMCR) is the monopolist incumbent until 2000 and Keurig is the company that holds the patent for producing k-cups ${ }^{3}$, in 2001 Keurig sold four licenses before GMCR fully acquired Keurig preventing additional sale of licenses (GMCR started increasing his percentage ownership of Keurig starting from 2003 and full acquisition is made in 2006). Interestingly GMCR then acquired (2009-2010) the

\footnotetext{
${ }^{2}$ We show that the incumbent is always best off delaying the bargaining process. This is not that obvious since on the one hand the later an agreement is reached, the lower is the remaining value of the patent (hence the lower is the price of the IP). But on the other hand since M could sell additional licenses after purchasing the IP, the earlier an agreement is reached the earlier he collects the additional entrants' profit.

${ }^{3} \mathrm{GMCR}$ is the first licensee since 1993.
} 
other four licensees to maintain monopoly power.

The idea that an action prior to the bargaining phase may serve as a commitment device to increase one party's bargaining position has been described in the context of incomplete contract and it is known as the hold-up problem (Williamson (1979) and Klein, Crawford, and Alchian (1978) ). In procurement relationship between two parties in which one party can make a relation-specific investment which can benefit both of them, this party may refrain from making such investment because of concerns that this may give the other party increased bargaining power. This can happen when the parties cannot sign an ex-ante complete and binding contract and it results with underinvestment in relation-specific investment and leads to inefficient outcome. Spiegel (1996) shows that a firm's incentive to make a relationspecific investment is related to the way these investments are financed. In particular, issuing debt to a third party may strengthens the firm's incentive to make relationship-specific investment, and therefore alleviates the hold-up problem. Diverting from this literature, we show that even under complete contract environment, in the context of patent licensing, the bargaining outcome may still be ex-ante inefficient. Schelling (1980) argues that an agent who can commit to pay to a third party contingent on his ex-post bargaining action may change his disagreement payoff and thereby increase his share in the "cake" (this idea was later formalized by Green (1991)). In our paper, commitments can be made only through licensing contracts and the terms are limited to the ownership of the IP, the licensing fee and the total number of licenses.

Our paper is also related to Hoppe, Jehiel, and Moldovanu (2006), who show that in case there are multiple incumbent firms (and many potential entrants) and the innovator sells licenses by auction, even though incumbents are driven by entry preemption motives and are willing to pay more for new licenses, sometimes entry occurs. Each incumbent is willing to avoid entry, but would rather prefer that the other incumbent pays the price of preemption. As a result, sometimes an entrant acquires the license because each incumbent is relying on the other to prevent entry. In their paper, in case the pre-innovation market is monopolistic, 
entry does not occur. We show that in case the innovator sells licenses sequentially (instead of via simultaneous auction), even if the market is initially a monopoly, entry may occur.

The next section (Section 2) presents in a general set-up a model of "co-opetition". Namely, a mixture of a bargaining between an outside innovator and incumbent monopoly (the cooperation part) and a competition between the incumbent and new entrant licensees. While the innovation has only a destructive value to the monopolist it has positive value to both innovator and society. The post-innovation market structure and the private value of the innovation are analyzed. The section provides conditions which guarantee entry to the market prior to the bargaining phase. In other words, conditions that guarantee that the innovation is not put on the shelf. It is shown that whenever the innovator has relatively weak bargaining power (e.g. a small lab versus a giant incumbent), the innovator is best off selling licenses to new entrants before bargaining with the incumbent firm. The loss from the small share of a shrinking "cake" is compensated by the change of the disagreement point to the advantage of the innovator and from the license fees collected during the bargaining phase. Similar result is obtained if the bargaining stage is expected to last for a significant period of time.

Section 3 deals with a special case where firms compete à la Cournot, first with a linear demand and then with a log-linear demand (with elasticity 1). The linear demand case is thoroughly analyzed and it is shown that the innovator may sell zero, one or two licenses (depending on the intensity of demand and his bargaining power) prior to the bargaining stage. Furthermore, after purchasing the IP the incumbent may find it beneficial to sell additional licenses. In addition we provide some examples where entry occurs, with loglinear demand.

Finally, even though the paper assumes that the innovator can meet the incumbent only once, the basic results hold even if the innovator has the opportunity to approach the incumbent more than once. Suppose that the innovator is allowed to meet the incumbent for a second time with high probability. Indeed, the innovator could explain to the incumbent 
in their first meeting and before selling any licenses what will happen if they fail to reach an agreement. Namely, the innovator then will sell a number of licenses before approaching the incumbent again causing a shrink of the bargaining cake. However, the incumbent who has every interest to stay with a monopoly power for as long as possible will delay the bargaining time to the limit, not only in the first round but also if he is approached again. As a result, it may still be beneficial for the innovator to sell a few licenses before approaching the incumbent for the first time. Moreover, if the incumbent in the first meeting with the innovator can make a credible commitment not to meet the innovator again if the bargaining fails, he will change the disagreement point in his favor. Taking this into account may also induce the innovator to sell some licenses before approaching the incumbent.

\section{The Model}

We consider a monopoly $(\mathrm{M})$ who produces a single good at a marginal $\operatorname{cost} c, c>0$. There are many potential entrants who are unable to enter the market under the existing technology which requires a high entry cost. An outside innovator (Inn) comes along with an alternative technology that eliminates the entry cost and with a marginal cost $c+\epsilon$ where $\epsilon \geq 0$. That is, the new technology is useless for $M$ as the marginal cost of production is no less than $c$. Yet M may purchase the IP of the new technology to limit subsequent entry. The length of the patent right is normalized to be 1 . Competition between $M$ and entrant licensees takes place along the whole period. The demand and the profits of all firms grow homogeneously in time. Denote by $\pi_{0}(k)$ and $\pi_{e}(k)$ the density profit of $\mathrm{M}$ and each entrant, respectively, when there are in total $k$ entrant licensees in the market. The profit of $\mathrm{M}$ and every entrant along $t, 0<t<1$, units of time is $t \pi_{0}(k)$ and $t \pi_{e}(k)$, respectively.

Assumption 1. (i) $\pi_{0}(k), \pi_{e}(k)$ and $\pi_{0}(k)+k \pi_{e}(k)$ are all decreasing in $k$. (ii) $\pi_{e}(1)>0$.

Assumption 1 (i) asserts that additional competition in the market reduces the total industry profit as well as the profit of the incumbent and each entrant licensee. As an 
example, Cournot competition with linear demand (or log-linear demand with elasticity 1) satisfies this assumption. Part (ii) asserts that Inn's new technology is useful for entrants. Namely, $\epsilon$ is not too larger.

Inn can sell a few licenses to new entrants (via a first-price auction) before bargaining with M over the IP of the new technology. Whatever the outcome of this bargaining is, the subsequent owner of the IP can sell additional licenses (via another first-price auction). If Inn decides to auction off some licenses before approaching $\mathrm{M}$, he announces a pair $(r, \delta)$ where $r>0$ is the number of licenses he sells to entrants in the current auction and $\delta$ is a commitment to sell no more than $\delta$ licenses in total. In the second auction the owner of the IP announces a number $j$ of additional licenses together with a commitment not to sell any more licenses. The number $j$ is bounded by previous commitment, namely $0 \leq j \leq \delta-r$.

If $r>0$, the first auction will take place at time 0 . Since there are a large number of potential entrants, because of the competition effect, we assume that the licenses will be sold immediately. That is, the first auction takes a negligible fraction of time. This is not the case in the bargaining between Inn and $M$ since there are no other incumbent firms in the market. It is assume that the bargaining between Inn and M can last at most $z$ units of time, $0<z<1$. Here $z$ captures an exogenously given deadline for a bargaining process which may be determined by social norm or industry convention. The bargaining, if Inn and $\mathrm{M}$ agree, can end at any time $t, 0 \leq t \leq z$.

The Pareto-frontier of the bargaining outcome consists of all divisions (between Inn and M) of the subsequent highest industry profit. Since the total industry profit is decreasing in the number of entrant licensees, if Inn approaches $M$ without selling any licenses, M after obtaining the IP will shelve it and the subsequent highest industry profit is the entire monopolist profit. If, however, Inn sells $r \geq 1$ licenses before approaching M, the optimal subsequent industry profit may be obtained by $\mathrm{M}$ selling additional number of licenses to new entrants. The disagreement payoffs are the payoffs of Inn and M following the failure of an agreement. In this case Inn may be best off selling additional licenses if it is feasible, 
namely if $\delta-r>0$. The bargaining solution must yield Inn a payoff, at least as high as the revenue he can collect from the subsequent auction in case the bargaining fails. This revenue is higher the sooner this auction takes place. Consequently, Inn is best off approaching M immediately after selling the first $r$ licenses to entrants. M, however, may have an opposite interest. This is not so obvious since M could also sell additional licenses after purchasing the IP and the earlier an agreement is reached, the earlier he collects the license fees from the additional entrant licensees. It is shown (Proposition 1, below) that nevertheless $\mathrm{M}$ is best off delaying the bargaining process as long as possible.

Suppose $r$ licenses are sold in the first auction. For convenience we allow $r=0$ (in which case first auction does not take place). Suppose an agreement is reached at time $t, 0 \leq t \leq z$. The subsequent optimal total industry profit to be shared by Inn and M is

$$
v(\delta, r)=(1-t)\left[m(\delta, r) \pi_{e}(m(\delta, r)+r)+\pi_{0}(m(\delta, r)+r)\right]
$$

where

$$
m(\delta, r)=\underset{0 \leq m \leq \delta-r}{\operatorname{argmax}}(1-t)\left[m \pi_{e}(m+r)+\pi_{0}(m+r)\right]
$$

is the optimal number of additional licenses $\mathrm{M}$ will sell if he obtains the IP.

The disagreement payoffs of Inn and M are

$$
d_{i n n}(\delta, r)=(1-t) \cdot n(\delta, r) \pi_{e}(r+n(\delta, r))
$$

and

$$
d_{M}(\delta, r)=(1-t) \cdot \pi_{0}(r+n(\delta, r))
$$

where

$$
n(\delta, r)=\underset{0 \leq n \leq \delta-r}{\operatorname{argmax}}(1-t) \cdot n \pi_{e}(r+n)
$$

is the number of additional licenses Inn will sell if the bargaining fails. Inn's disagreement 
payoff is the revenue he collects from the $n(\delta, r)$ additional licensees, and M's disagreement payoff is his profit when facing $r+n(\delta, r)$ competitors, both along $1-t$ units of time. Note that $m(\delta, r)$ and $n(\delta, r)$ do not depend on $t$. The next assumption assures that $n(\delta, r)$ and $m(\delta, r)$ are uniquely determined.

Assumption 2. For any $r, k \pi_{e}(r+k)$ and $k \pi_{e}(k+r)+\pi_{0}(k+r)$ are both single-peaked in $k$.

Given $(\delta, r)$, the bargaining problem is defined by $v(\delta, r)$ and $\left(d_{i n n}(\delta, r), d_{M}(\delta, r)\right)$. Inn and $M$ bargain over their shares in the surplus

$$
s(\delta, r)=v(\delta, r)-\left(d_{i n n}(\delta, r)+d_{M}(\delta, r)\right)
$$

By (1)-(6), $s(\delta, r) \geq 0$. Sharing the surplus depends on the (exogenously given) bargaining power $(\beta, 1-\beta)$ of Inn and $\mathrm{M}$, respectively (assuming that their relative bargaining power can be measured). The case where Inn has a small bargaining power compare with M may fit a scenario in which Inn is a small lab while $M$ is a giant incumbent. The "bargaining cake" is shared as follows:

$$
b_{i n n}(\delta, r)=\beta s(\delta, r)+d_{i n n}(\delta, r)
$$

and

$$
b_{M}(\delta, r)=(1-\beta) s(\delta, r)+d_{M}(\delta, r)
$$

The Nash bargaining solution is a special case where $\beta=\frac{1}{2}$. If $\beta=1$ Inn can make a take-it-or-leave-it offer and if $\beta=0$ it is $\mathrm{M}$ that can make such an offer. To avoid corner cases it is assumed that $\beta>0$. Namely the innovator has positive bargaining power.

Since the opportunity cost of an entrant is zero, anticipating the outcome of the bargaining, each one of the first $r$ licensees is willing to pay $t \pi_{e}(r)+(1-t) \pi_{e}(m(\delta, r)+r)$. Inn's 
total payoff is therefore

$$
\pi_{i n n}(\delta, r)=r\left[t \pi_{e}(r)+(1-t) \pi_{e}(m(\delta, r)+r)\right]+b_{i n n}(\delta, r)
$$

and M's total payoff is

$$
\pi_{M}(\delta, r)=t \pi_{0}(r)+b_{M}(\delta, r)
$$

Remark 1: $s(\delta, r)=0$ iff $m(\delta, r)=n(\delta, r)$ (by (6) and Assumption 2). In case $s(\delta, r)=0$, let $l \equiv m(\delta, r)=n(\delta, r)$. Inn's total payoff is $t \cdot r \pi_{e}(r)+(1-t) \cdot(l+r) \pi_{e}(l+r) \leq \max _{k} k \pi_{e}(k)$ and there is no gain from bargaining. Therefore it is assumed that in case $s(\delta, r)=0 \operatorname{Inn}$ will not bargain with M.

We can now define a strategic game $G$ between Inn and M. In the first stage Inn chooses and announces a pair $(\delta, r), \delta \geq r$. If $\delta=r$ or $s(\delta, r)=0$ (for $\delta>r$ ) Inn does not approach M. Inn obtains $r \pi_{e}(r), \mathrm{M}$ obtains $\pi_{0}(r)$ and the game ends. If $\delta>r$ and $s(\delta, r)>0$ the game proceeds to the second stage. Inn approaches $\mathrm{M}$ and $\mathrm{M}$ selects the bargaining time $t$, $0 \leq t \leq z$. In the third stage which lasts $1-t$ units of time, M purchases the IP and selects the number $m$ of additional licensees, where $m \leq \delta-r$. In any subgame perfect equilibrium of $G, m=m(\delta, r)$ and $n=n(\delta, r)$ must hold. The payoffs of Inn and M are therefore given by $(9)$ and $(10)$.

We first analyze the optimal choice of $m$ and $n$ if no previous restriction is imposed. Let the pair $(\infty, r)$ be the choice of Inn to sell $r$ licenses in the first auction with no restriction on future sale of licenses. Let $m(r) \equiv m(\infty, r)$ and $n(r) \equiv n(\infty, r)$. No confusion should result from this abuse of notation. That is, $m(r)(n(r))$ is the unconstraint optimal number of licenses M (Inn) will auction off in the second auction if an agreement is reached (no agreement is reached).

Lemma 1. For any $r, n(r)>m(r)$.

Proof. See A.1 of the Appendix. 
Lemma 1 states that Inn is better off selling more licenses than M would sell if he obtains the IP. The reason is simple. More licenses on one hand increases the incumbent's revenue from license fees but hurts his own profit, as a result of stronger competition. The latter effect does not apply to the innovator since he is an outsider.

Proposition 1. If Inn approaches $M$ an agreement will be reached only at the deadline, namely at $t=z$.

By Proposition 1 the first two stages of $G$ together last $z$ units of time and the third stage lasts $1-z$ units of time.

Proof. Let $m=m(\delta, r), n=n(\delta, r)$ and $s=s(\delta, r)$. Suppose Inn approaches $\mathrm{M}$ and an agreement is reached at time $t$. Prior to the agreement, $\mathrm{M}$ obtains $t \pi_{0}(r)$. The gross payoff of $\mathrm{M}$ after time $t$ is $(1-t)\left(m \pi_{e}(m+r)+\pi_{0}(m+r)\right)$ and the net payoff of $\mathrm{M}$ after $t$ is

$$
(1-t)\left(m \pi_{e}(m+r)+\pi_{0}(m+r)\right)-\overbrace{(1-t) \cdot\left(\beta s+n \pi_{e}(n+r)\right)}^{\text {Payment for the IP }} .
$$

Hence the total net payoff of $M$ is

$$
t \pi_{0}(r)+(1-t)\left(m \pi_{e}(m+r)+\pi_{0}(m+r)-\left(\beta s+n \pi_{e}(n+r)\right)\right) .
$$

Clearly this payoff is increasing in $t$ (implying $t=z$ ) if

$$
\pi_{0}(r)>m \pi_{e}(m+r)+\pi_{0}(m+r)-n \pi_{e}(n+r) .
$$

By Assumption $1 \pi_{0}(r) \geq \pi_{0}(m+r)$. We next show that $m \pi_{e}(m+r)<n \pi_{e}(n+r)$. By (5) $m \pi_{e}(m+r) \leq n \pi_{e}(n+r)$ and the equality holds iff $m(\delta, r)=n(\delta, r)$. By Remark 1 this happens iff $s(\delta, r)=0$, in which case Inn does not bargain with M, a contradiction. 
By Proposition 1, Inn maximizes over $\delta$ and $r$ his payoff $\pi_{i n n}(\delta, r)$ (given in (9p) for $t=z$. This payoff consists of (i) the license fees he obtains from the first $r$ competing licensees who also compete with $\mathrm{M}$ for $z$ units of time, (ii) the license fee Inn collects from the $r$ competing licensees who compete with $\mathrm{M}$ and with additional $m(\delta, r)$ licensees for the other $1-z$ units of time and (iii) Inn's share of the surplus $\beta s(\delta, r)$. By (1)-(9)

$$
\begin{aligned}
\pi_{i n n}(\delta, r)=(1-z) \cdot & \left(\beta\left(m \pi_{e}(r+m)+\pi_{0}(r+m)-n \pi_{e}(r+n)-\pi_{0}(r+n)\right)\right. \\
& \left.+n \pi_{e}(r+n)+r \pi_{e}(r+m)\right)+z \cdot r \pi_{e}(r)
\end{aligned}
$$

again where $m=m(\delta, r)$ and $n=n(\delta, r)$. We next analyze for any $r \geq 0$ the optimal commitment, $\delta$, of Inn.

Lemma 2. For any $r$, if $\delta \in[r, r+m(r)]$ then $s(\delta, r)=0$ and Inn does not benefit from bargaining with $M$.

Proof. Denote $l=\delta-r$. By Assumption $2, n(\delta, r)=\min (l, n(r))$ and $m(\delta, r)=\min (l, m(r))$. In case $l \leq m(r), m(\delta, r)=n(\delta, r)=l$. Lemma 2 then follows from Remark 1.

Remark 2: Setting $r \leq \delta \leq r+m(r)$ (in particular, $\delta=r$ ) on one hand limits the subsequent profit of M and shrinks the "bargaining cake", but on the other hand it allows Inn to increase the revenue he extracts from the first $r$ licensees, from $z r \pi_{e}(r)+(1-z) r \pi_{e}(r+$ $m(r))$ to $z r \pi_{e}(r)+(1-z) r \pi_{e}(\delta)$. Therefore, even though Inn cannot benefit from bargaining with $\mathrm{M}$ when setting $r \leq \delta \leq r+m(r)$ (Lemma 2), it is still possible that $\delta=r$ is optimal for him. The next Proposition shows that indeed this may be an equilibrium outcome for large $z$.

Proposition 2. Suppose $(\delta, r)$ is an equilibrium strategy of Inn. Then (i) either $\delta=r=$ $\operatorname{argmax}_{k} k \pi_{e}(k)$ and Inn does not bargain with $M$ or $\delta \geq r+n(r)$ and Inn bargains with $M$. (ii) There exists $z^{c}, 0<z^{c} \leq 1$ such that for any $z \in\left(0, z^{c}\right), \delta \geq r+n(r)$ and bargaining takes place. 
Proof. (i) If Inn does not approach M, the optimal license fee is obtained by choosing $\delta=$ $r=\operatorname{argmax}_{k} k \pi_{e}(k)$. Let $l=\delta-r$ and $A=\max _{k} k \pi_{e}(k)$. Consider next Inn's payoff when he approaches M. By Lemma 2, Inn obtains less than $A$ if $0 \leq l \leq m(r)$. Hence this can't happen in equilibrium. We also show that $m(r)<l<n(r)$ is not an equilibrium outcome. Inn's total profit when $l \geq n(r)$ is

$$
\begin{aligned}
\pi_{i n n}(\delta, r)=(1- & z)(\overbrace{\beta\left[(m(r)+r) \pi_{e}(m(r)+r)+\pi_{0}(m(r)+r)\right]+(1-\beta) r \pi_{e}(m(r)+r)}^{\text {part 1 }} \\
& +\underbrace{(1-\beta) n(r) \pi_{e}(n(r)+r)-\beta \pi_{0}(n(r)+r)}_{\text {part } 2})+z \cdot r \pi_{e}(r) .
\end{aligned}
$$

If $m(r)<l<n(r)$, then $n(\delta, r)=l$ and $m(\delta, r)=m(r)$. By (12), the total profit of Inn is

$$
\begin{array}{r}
\hat{\pi}_{i n n}(r, l)=(1-z)(\overbrace{\beta\left[(m(r)+r) \pi_{e}(m(r)+r)+\pi_{0}(m(r)+r)\right]+(1-\beta) r \pi_{e}(m(r)+r)}^{\text {part 1 }} \\
+\underbrace{(1-\beta) l \pi_{e}(l+r)-\beta \pi_{0}(l+r)}_{\text {part } 3})+z \cdot r \pi_{e}(r) .
\end{array}
$$

Note that part 1 of $(14)$ is the same as part 1 of $(13)$. We claim that part 3 of $(14)$ is smaller than part 2 of $(13)$. The reason is that $k \pi_{e}(k+r)$ is maximized at $k=n(r)$, and $\pi_{0}(k+r)$ is decreasing in $k$. Therefore $m(r)<l<n(r)$ is not an optimal choice for Inn. Intuitively, moving from $l \geq n(r)$ to $m(r)<l<n(r)$, the license fee of the first $r$ entrants as well as the subsequent "bargaining cake" remain the same. But the disagreement point changes to the disadvantage of Inn (since the optimal number of additional licenses Inn sells if no agreement is reached is now restricted by $l$ ).

(ii) Suppose $\delta(r)=\infty$ for any $r \geq 0$. By (12), the payoff of Inn when choosing $r$ is

$$
E \equiv(1-z) \cdot B+z \cdot \overbrace{r \pi_{e}(r)}^{C},
$$


where for $m=m(r)$ and $n=n(r)$

$$
\begin{aligned}
B & \equiv n \pi_{e}(r+n)+\beta\left[\left(m \pi_{e}(r+m)+\pi_{0}(r+m)\right)-\left(n \pi_{e}(r+n)+\pi_{0}(r+n)\right)\right]+r \pi_{e}(r+m) \\
& >n \pi_{e}(r+n) .
\end{aligned}
$$

The inequality in $(16)$ holds because $\beta>0$. Let $\hat{r} \in \operatorname{argmax}_{r} B$. Then

$$
\left.B\right|_{r=\hat{r}} \geq\left. B\right|_{r=0}>n(0) \pi_{e}(n(0))=A \geq\left. C\right|_{r=\hat{r}}
$$

Since $\left.B\right|_{r=\hat{r}}>A$, by $15|E|_{r=\hat{r}}>A$ iff $z<\left.\left(\frac{B-A}{B-C}\right)\right|_{r=\hat{r}}$. Since $\left(\max _{r} E\right) \geq\left. E\right|_{r=\hat{r}},\left(\max _{r} E\right)>$ $A$ for $z<\left.\left(\frac{B-A}{B-C}\right)\right|_{r=\hat{r}} \equiv z^{c}$.

Proposition 2 states that in some cases Inn is best off selling in total $r$ licenses, $r=$ $\operatorname{argmax}_{k} k \pi_{e}(k)$, and without bargaining with $\mathrm{M}$. This may happen only if the bargaining time $z$ is relatively large. Otherwise, bargaining with M benefits Inn. In this case any nonrestrictive $\delta, \delta \geq r+n(r)$ (including $\delta=\infty$ ), is optimal. Note that in case Inn is best off $n o t$ approaching $\mathrm{M}$, he sells positive number of licenses to entrants and the innovation is put to work.

Suppose next $z<z^{c}$. In this case the optimal $\delta$ does not affect the second auction and without loss of generality let $\delta=\infty$. Consistent with previous notations we omit $\delta$ and write $\pi_{i n n}(r)$ instead of $\pi_{i n n}(\delta, r)$, etc. Let $r^{*}$ be an equilibrium number of first auction licensees. Our next goal is to find sufficient conditions for $r^{*} \geq 1$.

Proposition 3. There exists $\bar{\beta}, 0<\bar{\beta}<1$, such that entry occurs for all $\beta, 0<\beta<\bar{\beta}$.

Proof. Let us first consider the case $r=0$. By $(12)$ since $z>0$

$$
\begin{aligned}
\lim _{\beta \rightarrow 0} \pi_{i n n}(\infty, 0) & =(1-z) n(0) \pi_{e}(n(0)) \\
& <n(0) \pi_{e}(n(0))=\pi_{i n n}(\delta=n(0), r=n(0))
\end{aligned}
$$

That is, when $\beta$ is sufficiently small, choosing $r=0$ (and bargaining with M) yields Inn a 
payoff less than selling $r=n(0) \geq 1$ licenses to entrants and without bargaining with $\mathrm{M}$. Proposition 3 follows from (17) and the continuity of $\pi_{i n n}(\infty, 0)$ with respect to $\beta$.

Proposition 3 asserts that entry occurs with certainty when Inn has a relatively weak bargaining power. In this case Inn obtains a small share of the "bargaining cake" and the loss from shrinking the cake is compensated by a change of the disagreement point to his advantage and from the license fees collected during the bargaining stage.

Suppose $0<\beta<\bar{\beta}$. Then by Proposition 3 entry occurs. If also $z<z^{c}$ then by Proposition 2 (ii) entry follows by Inn bargaining with M. If $z \geq z^{c}$ there are cases where Inn sells some licenses and does not approach M.

Proposition 4. Suppose $r^{*} \geq 1$ for $z=\hat{z}$. Then $r^{*} \geq 1$ for every $z, z>\hat{z}$.

Proof. For $\delta=\infty$ and for any $r \geq 0$ Inn's payoff can be written as

$$
\begin{aligned}
\pi_{i n n}(r)=(1-z) \cdot & {\left[\beta \cdot(\overbrace{(r+m) \pi_{e}(r+m)+\pi_{0}(r+m)}^{\hat{v}(r)}-(\overbrace{r \pi_{e}(r+m)+n \pi_{e}(r+n)}^{\hat{d}_{i n n}(r)})-\overbrace{\pi_{0}(r+n)}^{\hat{d}_{M}(r)})\right.} \\
& +\underbrace{r \pi_{e}(r+m)+n \pi_{e}(r+n)}_{\hat{d}_{i n n}(r)}]+z \cdot r \pi_{e}(r)
\end{aligned}
$$

where as before $m=m(r)$ and $n=n(r)$. By choosing $r=0$, Inn's payoff is

$$
\begin{aligned}
\pi_{i n n}(0)=(1-z) \cdot & {\left[\beta \cdot(\overbrace{\pi_{0}(0)}^{\hat{v}(0)}-\overbrace{n(0) \pi_{e}(n(0))}^{\hat{d}_{i n n}(0)}-\overbrace{\pi_{0}(n(0))}^{\hat{d}_{M}(0)})\right.} \\
& +\underbrace{n(0) \pi_{e}(n(0))}_{\hat{d}_{i n n}(0)}]+z \cdot 0
\end{aligned}
$$

By (18) and (19), for $r \geq 1, \pi_{i n n}(r)-\pi_{i n n}(0)>0$ iff

$$
\begin{aligned}
\left(\beta \cdot\left(\hat{v}(r)-\hat{d}_{i n n}(r)-\hat{d}_{M}(r)\right)+\hat{d}_{i n n}(r)\right)-\left(\beta \cdot\left(\hat{v}(0)-\hat{d}_{i n n}(0)-\hat{d}_{M}(0)\right)+\hat{d}_{i n n}(0)\right) & \\
& +\frac{z}{1-z} \cdot r \pi_{e}(r)>0 .
\end{aligned}
$$


For any $r$, the left-hand-side of 200 is increasing in $z$.

Proposition 4 states that Inn's willingness to sell a few licenses before approaching M is increasing with the bargaining time.

\section{$3 \quad$ Examples}

\subsection{Cournot Competition with Linear Demand}

In this section, it is assumed that firms are engaged in a Cournot competition and the inverse demand for the product is linear. Let $p=\max (a-Q, 0), a>c$, be the inverse demand and without loss of generality let $a-c=1$ (namely the per-period demand is 1 if $p=c)$. The profit functions of $\mathrm{M}$ and each entrant licensees satisfy both assumptions 1 and 2 (see A.2 of the Appendix).

Proposition 5. For any $z, 0<z \leq \frac{1}{2}$, Inn bargains with $M$ and chooses $\delta \geq r+n(r)$.

Proof. See A.3 of the Appendix.

We focus on $z \leq \frac{1}{2}$ and without loss of generality let $\delta=\infty$. The next Lemma states that Inn will sell in the first auction at most two licenses and hence maintain a relatively large "bargaining cake".

Lemma 3. $r^{*} \leq 2$.

Proof. See A.4 of the Appendix.

We first analyze Inn's optimal choice of $r$ for the limit case, $z \rightarrow 0$. For simplicity we focus on $r$ that is integer. Since $r^{*} \leq 2$ (by Lemma 3 we compare only the values $\pi_{i n n}(0)$, $\pi_{i n n}(1)$ and $\pi_{i n n}(2)$. The optimal choice of $r$ in case $z \rightarrow 0$ is summarized in Figure 1 (this numerical comparison is done using Maple. The original file is attached). Note that for 
$\epsilon \geq \frac{1}{2}$, the technology held by Inn is so inefficient that a licensee can make no profit. Entry will not occur in this case and the innovator obtains nothing from his patent. We therefore focus only on $\epsilon \in\left[0, \frac{1}{2}\right)$.

Proposition 6. In the limit case $z \rightarrow 0$, (i) $r^{*}$ is (weakly) decreasing in $\beta$. (ii) $r^{*}$ is (weakly) increasing in $\epsilon$ for $0 \leq \epsilon \leq \frac{1}{8}$ and (weakly) decreasing in $\epsilon$ for $\frac{1}{8}<\epsilon<\frac{1}{2}$.

Proposition 6 (i) is consistent with Proposition 3. It asserts that when Inn has a bigger bargaining power, he is best off selling no licenses prior to the bargaining stage. Intuitively, when $\beta$ is large, Inn obtains a bigger share of the total "cake" therefore his benefit from introducing more competition (which changes the disagreement point to his advantage) does not compensate for the loss of shrinking the size of the "cake".

The effect of $\epsilon$ on $r^{*}$ is less obvious. When the technology is more efficient, on one hand selling a license before bargaining reduces more the size of the "bargaining cake", while it increases more the threat on M. Let us divide the range of $\epsilon$ into two: $I_{1}=\left[0, \frac{1}{8}\right]$ and $I_{2}=\left(\frac{1}{8}, \frac{1}{2}\right)$. For any $\epsilon \in I_{2}$, even if $r=1, \mathrm{M}$ after obtaining the IP will sell no additional licenses $4^{4}$. In this case the more efficient the new technology is, the higher is the benefit from bringing in an entrant before bargaining with $\mathrm{M}$ (therefore enables a more severe threat) and it increases more than the damage it inflicts on the "cake". Inn then has higher incentive to choose $r=1$. However, for $\epsilon \in I_{1}$, if $r=1, \mathrm{M}$ after obtaining the IP will sell additional number of licenses (and this number is larger the more efficient is the technology). Taking this into account, for $\epsilon \in I_{1}$, when the technology becomes more efficient the damage of bringing in an entrant before bargaining with M increases more than the increase in Inn's benefit from a stronger threat, and Inn is more willing to choose $r=0$.

We next analyze the optimal choice of $r$ when the bargaining length is significant. Denote $L=\left[0, \frac{1}{2}\right) \times(0,1]$.

Proposition 7. (i) For any $(\epsilon, \beta) \in L$, the optimal choice of $r$ is (weakly) increasing in $z$.

\footnotetext{
${ }^{4}$ By $\left.(24)\right), m(0)=0$ and $m(1)=\max (0,1-8 \epsilon)$.
} 
(ii) For any $z>0$, there exists $(\epsilon, \beta) \in L$ such that the innovator sells positive number of licenses before approaching the incumbent.

Proposition 7 is consistent with Proposition 4. Figure 2 shows the optimal choice of $r$ for $z \rightarrow 0, z=1 / 3$ and $z=1 / 2$.

\subsection{Cournot Competition with Log-Linear Demand}

In this section, the demand function is assumed to be log-linear with elasticity 1. That is, $\log (Q)=\log (\alpha)-\xi \log (p)$ where $\xi=1$. Such demand function can be generated by consumers with Cobb-Douglas utility function. A separate Maple file shows that Assumption (1) and (2) are satisfied. The general analysis for log-linear demand is cumbersome therefore we only study one example: $c=1$ and $\alpha=1.5$. Suppose $z \rightarrow 0$, namely the bargaining between Inn and M takes almost no time. Figure 3 compares Inn's payoff when choosing $r=0$ and $r=1$, under different $\epsilon$ and $\beta$. Consistent with Proposition 3 , for $\beta$ sufficiently small, entry occurs with certainty. 


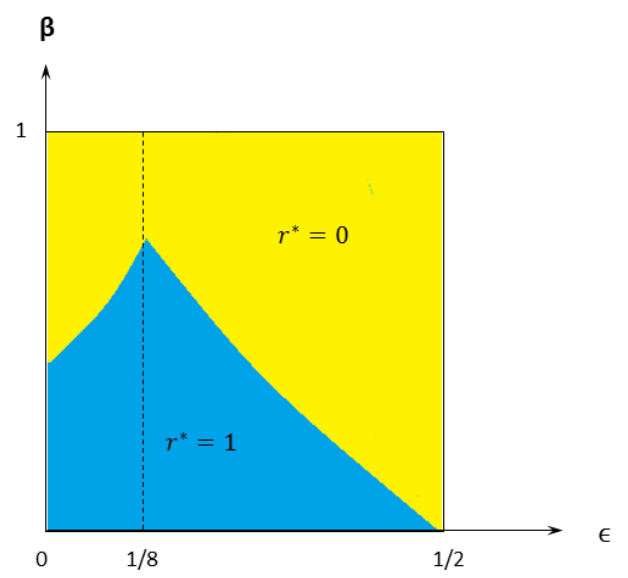

Figure 1: $r^{*}$ for $z \rightarrow 0$
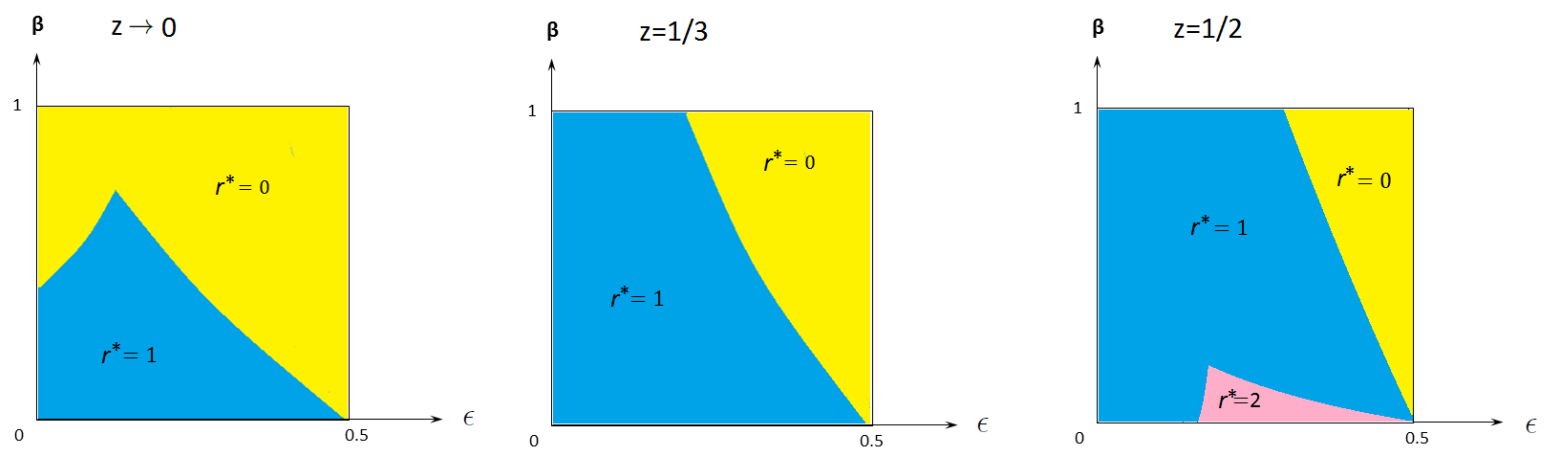

Figure 2 


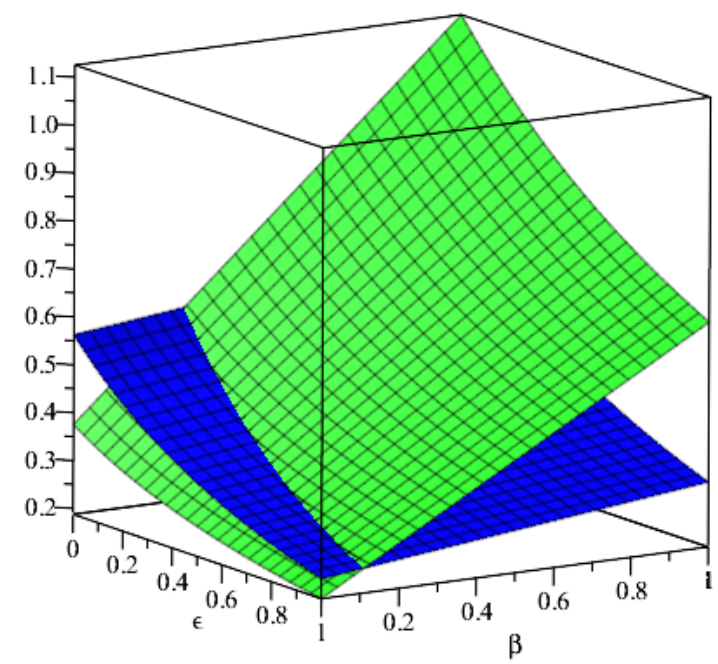

Figure 3: Comparison of Inn's Payoff at $r=0$ (green) and $r=1$ (blue)

\section{A Appendix}

\section{A.1 Proof of Lemma 1}

Recall that $n(r)=\operatorname{argmax}_{n \geq 0} n \pi_{e}(r+n)$ and $m(r)=\operatorname{argmax}_{m \geq 0}\left(m \pi_{e}(m+r)+\pi_{0}(m+\right.$ $r))$. By Assumption 2, $\left.\frac{\partial n \pi_{e}(r+n)}{\partial n}\right|_{n=n(r)}=0$ and $\left.\frac{\partial m \pi_{e}(m+r)}{\partial m}\right|_{m=m(r)}+\left.\frac{\partial \pi_{0}(m+r)}{\partial m}\right|_{m=m(r)}=$ 0 . Since $\pi_{0}(m+r)$ is decreasing in $m$ (Assumption 1), $\frac{\pi_{0}(m+r)}{\partial m}<0$. This implies that $\left.\frac{\partial m \pi_{e}(m+r)}{\partial m}\right|_{m=m(r)}>0$. Since $k \pi_{e}(r+k)$ is single-peaked in $k$ (Assumption 2), $m(r)<n(r)$.

\section{A.2 Cournot Competition with Linear Demand Satisfies Assump- tions 1 and 2}

Under Cournot competition with linear demand, $\pi_{0}(k)=\left(\frac{1+k \epsilon}{k+2}\right)^{2}$ and $\pi_{e}(k)=\left(\frac{1-2 \epsilon}{k+2}\right)^{2}$. When $\epsilon \geq \frac{1}{2}$, the new technology is so inefficient that entrants can make 0 profit. We focus only on the case $\epsilon<\frac{1}{2}$. Clearly $\pi_{e}(k)$ is decreasing in $k$. Since

$$
\frac{\partial \pi_{0}(k)}{\partial k}=\frac{2(k \epsilon+1)(2 \epsilon-1)}{(k+2)^{3}}
$$


and

$$
\frac{\partial\left(\pi_{0}(k)+k \pi_{e}(k)\right)}{\partial k}=\frac{(2 \epsilon-1)(4 \epsilon+k)}{(k+2)^{3}},
$$

for $\epsilon<\frac{1}{2}, \pi_{0}(k)$ and $\pi_{0}(k)+k \pi_{e}(k)$ are also decreasing in $k$. Assumption 1 follows.

Since

$$
\frac{\partial k \pi_{e}(r+k)}{\partial k}=\frac{(1-2 \epsilon)^{2}(r+2-k)}{(r+2+k)^{3}}
$$

and

$$
\frac{\partial\left(k \pi_{e}(r+k)+\pi_{0}(r+k)\right)}{\partial k}=\frac{(1-2 \epsilon)(r-4 r \epsilon-4 \epsilon-k)}{(r+2+k)^{3}},
$$

Assumption 2 follows.

\section{A.3 Proof of Proposition 5}

By Proposition 2, it is sufficient to show that $\max _{r} \pi_{i n n}(r)>\max _{k} k \pi_{e}(k)$ for $0<z \leq \frac{1}{2}$ $\left(\pi_{i n n}(r)\right.$ as defined in $(18)$. Let $A=\max _{k} k \pi_{e}(k)$. We next show that $\pi_{i n n}(1)>A$ for $0<z \leq \frac{1}{2}$. By 23$), k \pi_{e}(k)$ is maximized at $k=2$. Therefore

$$
A=(1-z) \cdot 2 \pi_{e}(2)+z \cdot 2 \pi_{e}(2) .
$$

By (18),

$$
\pi_{i n n}(1)=(1-z) \cdot B+z \cdot \pi_{e}(1)
$$

where $B=\beta\left(\hat{v}(1)-\hat{d}_{i n n}(1)-\hat{d}_{M}(1)\right)+\hat{d}_{i n n}(1)$.

We first compare the value of $B$ and $2 \pi_{e}(2)$. Since

$$
B-2 \pi_{e}(2)= \begin{cases}\frac{1}{144}(1-2 \epsilon)(20 \beta \epsilon+2 \beta-6 \epsilon+3) & 0 \leq \epsilon \leq \frac{1}{14} \\ \frac{1}{72}(1-2 \epsilon)(24 \beta \epsilon-10 \epsilon+5) & \frac{1}{14}<\epsilon \leq \frac{1}{2}\end{cases}
$$

it is easy to verify that $B>2 \pi_{e}(2)$ for $0 \leq \epsilon<\frac{1}{2}$. Note that $\pi_{e}(1)<2 \pi_{e}(2)$, thus by 25 and (26), $\pi_{i n n}(1)-A$ is decreasing in $z$. Therefore, to show that $\pi_{i n n}(1)>A$ for $0<z \leq \frac{1}{2}$, 
it is sufficient to show that $\left.\left(\pi_{i n n}(1)-A\right)\right|_{z=\frac{1}{2}}>0$. This indeed holds since

$$
\left.\left(\pi_{i n n}(1)-A\right)\right|_{z=\frac{1}{2}}= \begin{cases}\frac{1}{144}(1-2 \epsilon)(20 \beta \epsilon+2 \beta-2 \epsilon+1) & 0 \leq \epsilon \leq \frac{1}{14} \\ \frac{1}{18}(1-2 \epsilon)(6 \beta \epsilon-2 \epsilon+1) & \frac{1}{14}<\epsilon \leq \frac{1}{2}\end{cases}
$$

\section{A.4 Proof of Lemma 3}

By $(12)$, for $\delta=\infty$,

$$
\begin{aligned}
\pi_{i n n}(r)=(1 & -z)(\overbrace{\beta\left[(m(r)+r) \pi_{e}(m(r)+r)+\pi_{0}(m(r)+r)\right]+(1-\beta) r \pi_{e}(m(r)+r)}^{\mathrm{x}} \\
& +\underbrace{(1-\beta) n(r) \pi_{e}(n(r)+r)-\beta \pi_{0}(n(r)+r)}_{\mathrm{Y}})+z \cdot \underbrace{r \pi_{e}(r)}_{\mathrm{C}} .
\end{aligned}
$$

Note first that $C$ is single peaked in $r$ and maximized at $r=2$. It is then sufficient to show that $X+Y$ is maximized at $r \leq 2$.

It is easy to verify that $m(r)=\max (r(1-4 \epsilon)-4 \epsilon, 0)$. Expanding $X$ and $Y$,

$$
X+Y= \begin{cases}E & r(1-4 \epsilon)-4 \epsilon \geq 0 \\ F & r(1-4 \epsilon)-4 \epsilon<0\end{cases}
$$

where

$E=\frac{\left(4 \beta \epsilon^{2}+4 \epsilon^{2}-4 \epsilon+2\right) r^{3}+\left(12 \beta \epsilon^{2}+4 \beta \epsilon+16 \epsilon^{2}-16 \epsilon+8\right) r^{2}+\left(12 \beta \epsilon^{2}+8 \beta \epsilon+20 \epsilon^{2}+\beta-20 \epsilon+9\right) r+4 \beta \epsilon^{2}+4 \beta \epsilon+8 \epsilon^{2}+\beta-8 \epsilon+2}{4(r+2)^{2}(1+r)^{2}}$

and

$$
F=-\frac{\left(12 \beta \epsilon^{2}-8 \beta \epsilon-20 \epsilon^{2}+\beta+20 \epsilon-5\right) r+12 \beta \epsilon^{2}-4 \beta \epsilon-8 \epsilon^{2}-\beta+8 \epsilon-2}{4(r+2)^{2}}
$$


Note that $X+Y$ is continues in $r$. We first analyze the effect of $r$ on the value of $E$.

$$
\frac{\partial E}{\partial r}=-\frac{\left(4 \beta \epsilon^{2}+4 \epsilon^{2}-4 \epsilon+2\right) r^{4}+\left(12 \beta \epsilon^{2}+8 \beta \epsilon+20 \epsilon^{2}-20 \epsilon+10\right) r^{3}+\left(12 \beta \epsilon^{2}+24 \beta \epsilon+36 \epsilon^{2}+3 \beta-36 \epsilon+15\right) r^{2}+\left(4 \beta \epsilon^{2}+24 \beta \epsilon+28 \epsilon^{2}+7 \beta-28 \epsilon+3\right) r+8 \beta \epsilon+8 \epsilon^{2}+4 \beta-8 \epsilon-6}{4(r+2)^{3}(1+r)^{3}} .
$$

Rewrite (31):

$$
\frac{\partial E}{\partial r}=-\frac{E_{4} r^{4}+E_{3} r^{3}+E_{2} r^{2}+E_{1} r+E_{0}}{4(r+2)^{3}(1+r)^{3}},
$$

where $E_{4}=4 \beta \epsilon^{2}+4 \epsilon^{2}-4 \epsilon+2, E_{3}=12 \beta \epsilon^{2}+8 \beta \epsilon+20 \epsilon^{2}-20 \epsilon+10, E_{2}=12 \beta \epsilon^{2}+24 \beta \epsilon+$ $36 \epsilon^{2}+3 \beta-36 \epsilon+15, E_{1}=4 \beta \epsilon^{2}+24 \beta \epsilon+28 \epsilon^{2}+7 \beta-28 \epsilon+3$ and $E_{0}=8 \beta \epsilon+8 \epsilon^{2}+4 \beta-8 \epsilon-6$.

Clearly, for $0 \leq \epsilon<\frac{1}{2}, E_{4}>0$ and $E_{3}>0$. Consider next $E_{2} r^{2}+E_{1} r+E_{0}$. Since

$$
E_{2} \geq 36 \epsilon^{2}-36 \epsilon+15>0
$$

,$E_{2} r^{2}+E_{1} r+E_{0}$ reaches the minimum at $r=-\frac{E_{1}}{2 E_{2}}$. Since

$$
4 E_{2}+E_{1}=52 \beta \epsilon^{2}+120 \beta \epsilon+172 \epsilon^{2}+19 \beta-172 \epsilon+63>0,
$$

$-\frac{E_{1}}{2 E_{2}}<2$ and $E_{2} r^{2}+E_{1} r+E_{0}$ is increasing in $r$ for $r \geq 2$. Since

$$
\left.\left(E_{2} r^{2}+E_{1} r+E_{0}\right)\right|_{r=2}=56 \beta \epsilon^{2}+152 \beta \epsilon+208 \epsilon^{2}+30 \beta-208 \epsilon+60>0,
$$

$E_{2} r^{2}+E_{1} r+E_{0}>0$ for $r \geq 2$. As a result, for $r \geq 2, \frac{\partial E}{\partial r}<0$ and $E$ is decreasing in $r$.

Consider next the effect of $r$ on the value of $F$.

$$
\frac{\partial F}{\partial r}=\frac{\left((12 \beta-20) \epsilon^{2}+(-8 \beta+20) \epsilon+\beta-5\right) r+8 \beta \epsilon+24 \epsilon^{2}-4 \beta-24 \epsilon+6}{4(r+2)^{3}}
$$

It can be verified that $(12 \beta-20) \epsilon^{2}+(-8 \beta+20) \epsilon+\beta-5<0$ for $0<\beta<1$ and $0 \leq \epsilon<\frac{1}{2}$. When $r=2$,

$$
\frac{\partial F}{\partial r}=-\frac{1}{128}(1-2 \epsilon)(6 \beta \epsilon+\beta+2-4 \epsilon)<0 .
$$


Therefore for $r \geq 2, \frac{\partial F}{\partial r}<0$ and $F$ is decreasing in $r$.

Since both $E$ and $F$ are decreasing in $r$ for $r \geq 2, X+Y$ is decreasing in $r$ for $r \geq 2$.

\section{References}

[1] Richard J Gilbert and David MG Newbery. "Preemptive patenting and the persistence of monopoly". In: The American Economic Review (1982), pp. 514-526.

[2] Jerry R Green. "10 The Strategic Use of Contracts with Third Parties". In: Strategy and Choice (1991), p. 241.

[3] Heidrun C Hoppe, Philippe Jehiel, and Benny Moldovanu. "License auctions and market structure". In: Journal of Economics \& Management Strategy 15.2 (2006), pp. 371-396.

[4] Benjamin Klein, Robert G Crawford, and Armen A Alchian. "Vertical integration, appropriable rents, and the competitive contracting process". In: The Journal of Law $\mathcal{E}$ Economics 21.2 (1978), pp. 297-326.

[5] Thomas Schelling. The Strategy of Conflict. Harvard university press, 1980.

[6] Yossef Spiegel. "The role of debt in procurement contracts". In: Journal of Economics 86 Management Strategy 5.3 (1996), pp. 379-407.

[7] Oliver E Williamson. "Transaction-cost economics: the governance of contractual relations". In: The Journal of Law \& Economics 22.2 (1979), pp. 233-261. 